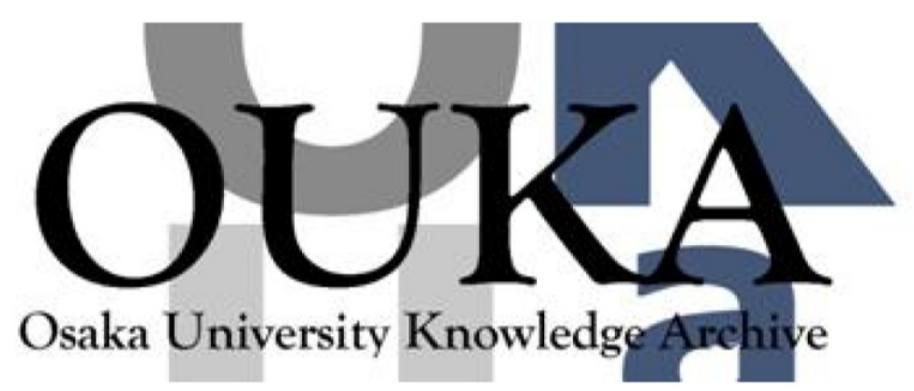

\begin{tabular}{|c|l|}
\hline Title & $\begin{array}{l}\text { Study of ultraintense laser propagation in } \\
\text { overdense plasmas for fast ignition }\end{array}$ \\
\hline Author(s) & Lei, A.L.; Tanaka, K. A. ; Kodama, R. et al. \\
\hline Citation & Physics of Plasmas. 16(5) p.056307 \\
\hline Issue Date & $2009-03$ \\
\hline oaire:version VoR \\
\hline URL & https://hdl. handle. net/11094/3031 \\
\hline rights & \\
\hline Note & \\
\hline
\end{tabular}

Osaka University Knowledge Archive : OUKA

https://ir. Library. osaka-u. ac. jp/

Osaka University 


\title{
Study of ultraintense laser propagation in overdense plasmas for fast ignition ${ }^{a)}$
}

\author{
A. L. Lei, ${ }^{1,2, b)}$ K. A. Tanaka, ${ }^{2}$ R. Kodama,${ }^{2}$ K. Adumi, ${ }^{2}$ H. Habara,${ }^{2}$ Y. Kitagawa, ${ }^{2}$ \\ K. Kondo, ${ }^{2}$ T. Matsuoka, ${ }^{2, c}$ T. Tanimoto, ${ }^{2}$ T. Yabuuchi, ${ }^{2, d)}$ K. Mima, ${ }^{3}$ K. Nagai, ${ }^{3}$ \\ H. Nagatomo, ${ }^{3}$ T. Norimatsu, ${ }^{3}$ K. Sawai, ${ }^{3}$ K. Suzuki, ${ }^{3}$ Wei Yu, ${ }^{1}$ Han Xu, ${ }^{1}$ X. Q. Yang, ${ }^{1}$ \\ L. H. Cao, ${ }^{4}$ H. B. Cai, ${ }^{3,4}$ Y. Sentoku, ${ }^{5}$ A. Pukhov, ${ }^{6}$ R. Kumar, ${ }^{7}$ R. Snavely, ${ }^{8}$ \\ R. Freeman, ${ }^{9}$ Min Yu, ${ }^{10}$ and J. Zheng ${ }^{11}$ \\ ${ }^{1}$ Shanghai Institute of Optics and Fine Mechanics, Chinese Academy of Sciences, Shanghai 201800, China \\ ${ }^{2}$ Graduate School of Engineering and Institute of Laser Engineering, Osaka University, \\ Suita, Osaka 565-0871, Japan \\ ${ }^{3}$ Institute of Laser Engineering, Osaka University, Suita, Osaka 565-0871, Japan \\ ${ }^{4}$ Institute of Applied Physics and Computational Mathematics, Beijing 100088, China \\ ${ }^{5}$ Department of Physics, NTF-MS372, University of Nevada, Reno, Nevada 89506, USA \\ ${ }_{7}^{6}$ Institut fur Theoretische Physik I, Heinrich-Heine-Universitat Duesseldorf, Duesseldorf 40225, Germany \\ ${ }^{7}$ Tata Institute of Fundamental Research, Mumbai 400005, India \\ ${ }^{8}$ Lawrence Livermore National Laboratory, University of California, Livermore, California 94550, USA \\ ${ }^{9}$ College of Mathematical and Physical Sciences, Ohio State University, \\ Columbus, Ohio 43210, USA \\ ${ }^{10}$ Institute for Fusion Theory and Simulation, Zhejiang University, Hangzhou 310027, China \\ ${ }^{11}$ Department of Modern Physics, University of Science and Technology of China, \\ Hefei, Anhui 230026, China
}

(Received 5 December 2008; accepted 27 February 2009; published online 8 April 2009)

\begin{abstract}
Laser plasma interactions in a relativistic regime relevant to the fast ignition in inertial confinement fusion have been investigated. Ultraintense laser propagation in preformed plasmas and hot electron generation are studied. The experiments are performed using a $100 \mathrm{TW} 0.6 \mathrm{ps}$ laser and a $20 \mathrm{TW}$ 0.6 ps laser synchronized by a long pulse laser. In the study, a self-focused ultraintense laser beam propagates along its axis into an overdense plasma with peak density $10^{22} / \mathrm{cm}^{3}$. Channel formation in the plasma is observed. The laser transmission in the overdense plasma depends on the position of its focus and can take place in plasmas with peak densities as high as $5 \times 10^{22} / \mathrm{cm}^{3}$. The hot electron beams produced by the laser-plasma interaction have a divergence angle of $\sim 30^{\circ}$, which is smaller than that from laser-solid interactions. For deeper penetration of the laser light into the plasma, the use of multiple short pulse lasers is proposed. The latter scheme is investigated using particle-in-cell simulation. It is found that when the pulse duration and the interval between the pulses are appropriate, the laser pulse train can channel into the plasma deeper than a single longer pulse laser of similar peak intensity and total energy. (C) 2009 American Institute of Physics.
\end{abstract}

[DOI: $10.1063 / 1.3101912$ ]

\section{INTRODUCTION}

Controlled fusion energy driven by high-power lasers has been investigated since the invention of the laser. In the conventional approach to inertial confinement fusion, a small volume deuterium-tritium (DT) fuel (the fuel core) is compressed and heated simultaneously by shock waves from the laser driven implosion of a spherical shell containing the fuel. To achieve energy gain the laser energy should be more than $1 \mathrm{MJ}$ and applied within a few nanoseconds. In principle, such a fuel core can be prepared by performing the compression and heating separately. In the fast ignition (FI)

\footnotetext{
a) Paper YI1 6, Bull. Am. Phys. Soc. 53, 321 (2008).

${ }^{b)}$ Invited speaker.

${ }^{c)}$ Present address: FOCUS Center and Center for Ultrafast Optical Science, University of Michigan, Ann Arbor, MI 48109, USA.

${ }^{d)}$ Present address: Center for Energy Research, University of California, San Diego, CA 92093, USA.
}

scheme, ${ }^{1-3}$ the required ultrahigh fuel density is achieved via conventional laser driven implosion, but the ultrahigh ignition temperature is achieved via fast heating by a separate ultrashort ultraintense (USUI) laser pulse. The FI scheme significantly reduces the total laser energy required and thus also the size of the fusion facility. However, to achieve FI the USUI laser must propagate into the compressed fuel core through the corona plasma that contains underdense as well as overdense regions. One solution is to insert a hollow cone in the fuel shell to block the USUI laser from the coronal plasma, thus allowing it to reach the core plasma. ${ }^{2}$ This method is effective in heating the core plasma and increasing the thermal neutron yield. However, the cone-in-shell geometry precludes the more symmetrical high-gain target designs. An alternative approach is to inject USUI laser pulses directly into the coronal plasma. ${ }^{3}$ Large numbers of hot electrons with energies in the $\mathrm{MeV}$ range are generated and transported into the core plasma. For efficient heating, the hot electron beam should be well collimated and the distance 
from its source to the core plasma should be minimized. That is, the USUI heating laser should propagate as deep as possible into the plasma.

Ultraintense laser propagation in plasmas has been explored extensively. ${ }^{4-15}$ When the laser power exceeds the critical value $P_{c r}=17 n_{c} / n_{e} \mathrm{GW}$, where $n_{e}$ and $n_{c}$ are the plasma and critical densities, respectively, it can undergo relativistic self-focusing (RSF). ${ }^{4}$ The laser pulse can propagate in the overdense plasma because of relativistic induced transparency ${ }^{5}$ (RIT) and laser hole boring (LHB). ${ }^{6}$ RIT allows the laser to propagate in a plasma with density less than $\gamma n_{c}$, where $\gamma=\sqrt{1+\left[I \lambda^{2} /\left(2.74 \times 10^{18}\right)\right]}$ is the Lorenz factor, $I$ is the laser intensity in $\mathrm{W} / \mathrm{cm}^{-2}$, and $\lambda$ is the laser wavelength in microns. RIT with RSF can induce self-guiding of the ultraintense laser in high-density plasmas. Tanaka and co-workers ${ }^{14,15}$ observed a propagation mode (the superpenetration mode) involving RSF, RIT, and LHB. When a subpicosecond $100 \mathrm{TW}$ laser is injected into a preformed plasma, strong x-ray emission around the solid-target surface was observed, indicating that the laser pulse can propagate $100 \mu \mathrm{m}$ in the inhomogeneous overdense plasma with densities up to that of solid.

In this work, we consider superpenetration of short ultraintense lasers in preformed plasmas relevant to FI using a laser system consisting of 100 and 20 TW laser. Particlein-cell (PIC) simulation is used to consider the use of multiple USUI lasers to improve the penetration depth. We shall focus on the laser beam behavior in the plasma as well as hot electron generation. Accordingly, plasma channel formation, laser light transmission, hot electron energy spectra, and the electron beam divergence are investigated.

\section{EXPERIMENTAL SETUP}

The experiments were conducted with the $100 \mathrm{TW}$ (GEKKO PW) (Ref. 16) and the 20 TW (GEKKO MII) (Ref. 17) short pulse laser systems coupled by a long pulse laser from the Institute of Laser Engineering, Osaka University. The GEKKO PW pulse can deliver 100-250 J with 0.6 ps duration at $1 \mu \mathrm{m}$ wavelength. The focus spot size was about $70 \mu \mathrm{m}$, with intensity $0.65-1.1 \times 10^{19} \mathrm{~W} / \mathrm{cm}^{2}$. The preformed plasmas were created with thin deuterated carbon (CD) foils with $\mathrm{Al}$ coating on the front surface by focusing a beam of the long pulse $0.53 \mu \mathrm{m}$ GEKKO XII laser. The plasma density profiles were controlled by changing the time delay between the GEKKO PW and GEKKO XII lasers. Typically, the peak density of the preformed plasma is around or higher than $10 n_{c}$. The GEKKO MII laser can deliver $6-10 \mathrm{~J}$ with $0.6 \mathrm{ps}$ duration at $1 \mu \mathrm{m}$ wavelength. The spot was elliptical with 13 and $33 \mu \mathrm{m}$ [full width at half maximum (FWHM)] for the axial and the transverse widths, respectively, giving an intensity of $1.6-2.4 \times 10^{18} \mathrm{~W} / \mathrm{cm}^{2}$. The preformed plasmas were created from thin $\mathrm{CD}$ foils with Al coated front surface by focusing a long pulse $1 \mu \mathrm{m}$ laser. The peak density of the plasma is up to $50 n_{c}$.

\section{EXPERIMENTS AND RESULTS}

We consider superpenetration of an ultraintense laser into the preformed plasma. Plasma channel formation, light transmission through the large scale inhomogeneous plasmas, and hot electron generation were investigated. The experiments were carried out using planar targets.

\section{A. Plasma channel formation}

In FI, the ultraintense laser energy must be transmitted into the core plasma through the thick coronal plasma which contains large underdense and overdense regions. For example, a CD shell target of $500 \mu \mathrm{m}$ diameter and $8 \mu \mathrm{m}$ shell thickness imploded by a $2 \mathrm{~kJ} 0.53 \mu \mathrm{m} 1 \mathrm{~ns}$ laser has a length over $500 \mu \mathrm{m}$ at a density $0.1-1 n_{c}$, and $200 \mu \mathrm{m}$ at $1 n_{c}$ to $10 n_{c} \cdot{ }^{3}$ In the context of FI, one important question is whether an ultraintense laser with an intensity $\sim 10^{19} \mathrm{~W} / \mathrm{cm}^{2}$ can penetrate the thick coronal plasma with entire beam self-focused. In an earlier experiment, Tanaka and co-workers ${ }^{14,15}$ observed strong $\mathrm{x}$-ray emission around the solid-target surface, which might indicate that the intense laser could propagate $100 \mu \mathrm{m}$ in inhomogeneous overdense plasmas into near solid regions. However, this x-ray emission can also be caused by the local heating of the solid surface by the hot electrons generated in the ultraintense laser propagation. These electrons may be generated far from the solid surface and they transport to the surface and heat it, causing $\mathrm{x}$-ray emission. It is desirable for FI to study the details of RSF behaviors in a preformed plasma with a large overdense region. We have studied laser light behaviors in the preformed plasma which has a $80 \mu \mathrm{m}$ overdense region and is created on a plane solid CD target.

The experiments were carried out with the $100 \mathrm{TW}$ GEKKO PW laser synchronized with the GEKKO XII laser system within a time jitter of less than $10 \mathrm{ps} .{ }^{18}$ Preformed plasmas were created by one beam of the $1-1.3 \mathrm{~ns}$ long pulse GEKKO XII laser with $1 \mu \mathrm{m}$ thick CD foils with $0.1 \mu \mathrm{m}$ Al coating on the front surface. The preformed plasma density is calculated using a one-dimensional (1D) hydrocode code ILESTA_1D. ${ }^{19}$ The ILESTA_1D has been benchmarked by $\mathrm{x}$-ray, ultraviolet, and optical laser probe diagnostics ${ }^{20,21}$ as well as used to characterize the plasma density profile. The typical plasma density profile at the time of the GEKKO PW laser injection has a length of $500 \mu \mathrm{m}$ at density of 0.1 to $1 n_{c}$, with an $80 \mu \mathrm{m}$ overdense region at a peak density of $10 n_{c}$. The uncertainty in the peak plasma density may come from the timing jitter between the GEKKO PW and GEKKO XII lasers. In the experiment, the GEKKO PW laser was optically synchronized to the GEKKO XII and the jitter was less than $10 \mathrm{ps}$. Within $10 \mathrm{ps}$, the hydrodynamic motion of the preformed plasma can be neglected. Figure 1 shows the time-integrated $\mathrm{x}$-ray images of the self-emission in front of the target at a view angle of $21^{\circ}$ from the GEKKO PW laser axis. Figures 1(a) and 1(b) correspond to the preformed plasma with and without the GEKKO PW laser interactions, respectively. The large $500 \mu \mathrm{m}$ emission areas shown as an ellipse in Figs. 1(a) and 1(b) are due to the long pulse GEKKO XII laser irradiation on the planar target. There is a distinct difference between Figs. 1(a) and 1(b). In Fig. 1(a), 

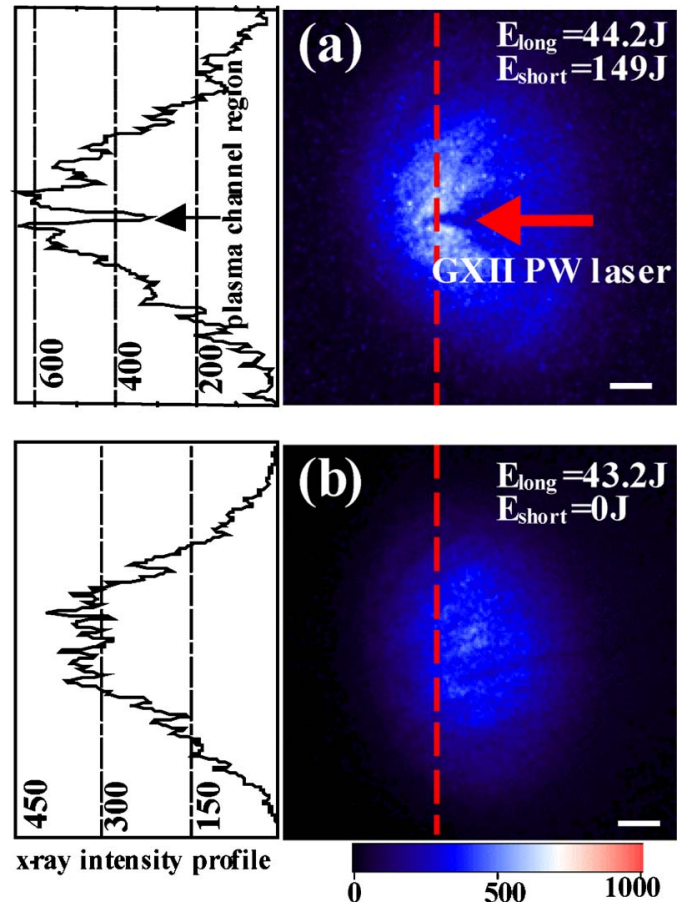

FIG. 1. (Color online) Time-integrated x-ray pinhole camera images from the preformed plasmas taken (a) with, and (b) without the GEKKO PW laser interactions (from Ref. 18). The x-ray intensity profiles along the vertical dashed lines in the images are at the left of the images. $E_{\text {long }}$ and $E_{\text {short }}$ denote the energies of the long pulse GEKKO XII laser and the short pulse GEKKO PW laser. The color codes are in arbitrary units. The white scale bars correspond to $100 \mu \mathrm{m}$. Note the $\mathrm{x}$-ray pictures were obtained at $21^{\circ}$ to the GEKKO PW laser axis (i.e., not $90^{\circ}$ ).

one sees an elongated cone-shaped region at the center of the ellipse. The x-ray emission from this region is considerably weaker than that from the surrounding region. The cone is along the GEKKO PW laser axis, indicating that it is created by the interaction of the GEKKO PW laser with the preformed plasma. The strong ponderomotive force drives the electrons and subsequently also the ions out of the target, leading to depletion of plasma particles and formation of a channel. After passage of the GEKKO PW laser and channel formation, the long pulse GEKKO XII laser still irradiates the entire preformed plasma for several $100 \mathrm{ps.} \mathrm{The} \mathrm{plasma}$ channel with depleted electrons and ions emits less x-ray than the rest of the plasma. The cone structure in the x-ray image is reproducible in the experiments and can be reconstructed with analytical estimates based on inverse bremsstrahlung x-ray emission and absorption with given plasma parameters. We have simultaneously measured the GEKKO PW laser transmission through the preformed plasma, which was approximately $4 \%$ of the laser energy. Therefore the $\mathrm{x}$-ray picture shown in Fig. 1(a) and the laser light transmission measurements indicate that the GEKKO PW laser penetrates through the entire preformed plasma having large underdense and overdense regions along the laser axis. Estimation of the GEKKO PW laser transmission taking into account RIT and LHB (Ref. 6) suggests that the GEKKO PW laser was self-focused down from $70 \mu \mathrm{m}$ to at least $20 \mu \mathrm{m}$ to penetrate through the preformed plasma in order to produce the observed light transmission.

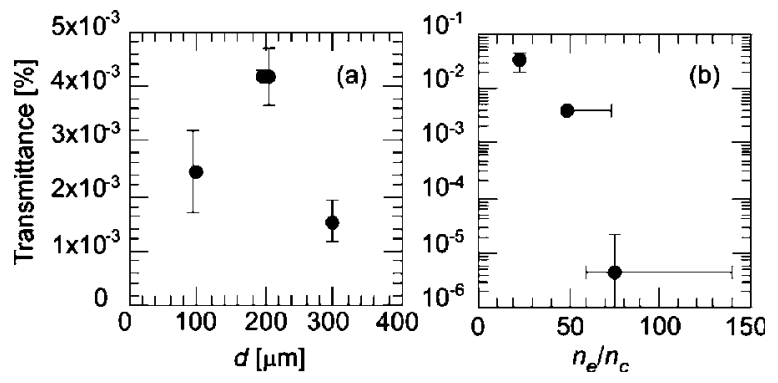

FIG. 2. Ultraintense laser transmission through the overdense plasmas as a function of (a) focal position at peak plasma density of $50 n_{c}$ and of (b) the peak density in the preformed plasma at fixed focal position $d=200 \mu \mathrm{m}$, respectively (from Ref. 22).

\section{B. Ultraintense laser transmission through the overdense plasmas}

In FI, the imploded plasma density profile starts to show a steep gradient around 20-50 $n_{c}$. Below this value the plasma density gradually increases. For example, a $2 \mathrm{~kJ}$ $0.53 \mu \mathrm{m} 1 \mathrm{~ns}$ laser driven imploded plasma from a CD shell target of $500 \mu \mathrm{m}$ diameter and $8 \mu \mathrm{m}$ shell thickness will have its density varying from 0.1 to $1 n_{c}$ in $500 \mu \mathrm{m}$, and from $20 n_{c}\left(50 n_{c}\right)$ to $1000 n_{c}$ in only $50 \mu \mathrm{m}(40 \mu \mathrm{m}){ }^{3}$ Therefore if the ultraintense laser can propagate into the 20-50 $n_{c}$ region and generate hot electrons there, the heating of the core plasma by the latter should be efficient since the remaining distance to the core is short.

The experiments were carried out with the 20 TW 0.6 ps GEKKO MII laser system coupled with a 400 ps long pulse laser. ${ }^{22}$ Preformed plasmas were created by the long pulse laser in $0.25-1 \mu \mathrm{m}$ thick CD foils whose front surface is coated with $0.1 \mu \mathrm{m}$ thick Al. The thickness of the CD layer was varied to control the peak density of the preformed plasma from 23 to $75 n_{c}$, corresponding to 0.25 to $1 \mu \mathrm{m}$ thick CD foils. In the experiments, the focus position $d$ of the GEKKO MII laser was varied 300-100 $\mu \mathrm{m}$ from the critical density. Figure 2 shows the laser light transmission through the preformed plasma. The error bar of the transmission is due to noise in the diagnostics. For fixed peak plasma density $50 n_{c}$, one can see that the transmission depends on $d$ [Fig. 2(a)] and it reaches maximum at $d=200 \mu \mathrm{m}$. For fixed focus position $d=200 \mu \mathrm{m}$, the laser transmission decreases as the plasma peak density is increased [Fig. 2(b)]. A transmission of $\sim 0.03 \%$ was obtained when the peak density was $23 n_{c}$. The dependence on the focus position was investigated earlier by Tanaka and co-workers ${ }^{14,15}$ using the $\mathrm{x}$-ray emission measurement. They found strong x-ray emission around the target surface only when the (100 TW) laser was focused at a certain position in the preformed plasma, suggesting that the laser propagates a long distance into the high-density region. The present experiment also shows such dependence on the focus position. In particular, there exists an optimum focus position for laser light transmission through the preformed plasma, as shown in Fig. 2(a).

The transmission of the GEKKO MII laser through the overdense plasma with a peak density of $23 n_{c}$ shows that there is strong self-focusing of the laser beam in the plasma. By using Sentoku's LHB velocity [Eq. (24) in Ref. 23], 

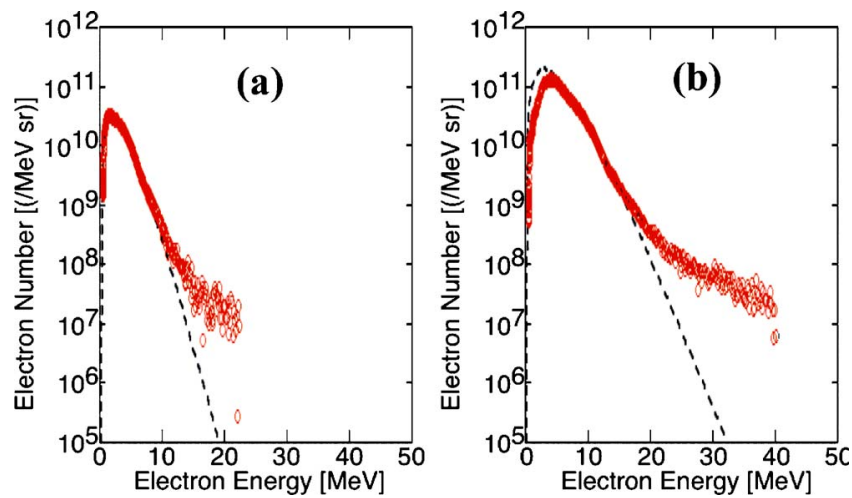

FIG. 3. (Color online) The electron energy spectra for (a) the small scale and (b) the large scale preformed plasma (from Ref. 24). The dotted lines are fitted curves with a relativistic Maxwellian distribution.

we can estimate that the required laser intensity for transmission through a plasma with peak density $23 n_{c}$ is $1.2 \times 10^{21} \mathrm{~W} / \mathrm{cm}^{2}$, which is nearly three orders of magnitude higher than the initial laser intensity. ${ }^{22}$

\section{Hot electron generation}

In FI, the core plasma is heated by the hot electrons generated during the ultraintense laser propagation in the large scale plasma. The heating efficiency depends on many factors, such as the energy and spatial distribution of the hot electrons. A collimated hot electron beam with appropriate energy range is essential for the efficient heating. Generation of hot electrons was investigated using 100 TW GEKKO PW laser. $^{24}$ The electron energy spectra were obtained using an electron spectrometer from $21^{\circ}$ to the laser axis. The electron angular distribution is obtained with a detector stack consisting of imaging plates ${ }^{25}$ placed $34 \mathrm{~mm}$ behind the target. Preformed plasmas were created by one beam of the $1-1.3 \mathrm{~ns}$ long pulse GEKKO XII laser and $1 \mu \mathrm{m}$ thick CD foils with $0.1 \mu \mathrm{m} \mathrm{Al}$ coating on the front surface. The plasma density profiles were controlled by the time delay between the short pulse GEKKO PW and the long pulse GEKKO XII lasers. That is, the GEKKO PW laser was injected into the preformed plasmas with either large or small underdense and overdense regions. The plasma scale length was only $3 \mu \mathrm{m}$ for the small plasma and $\sim 136 \mu \mathrm{m}$ for the large plasma. Figures 3(a) and 3(b) show the typical electron energy spectra from the small and large plasmas, respectively, at a laser intensity of $4 \times 10^{18} \mathrm{~W} / \mathrm{cm}^{2}$. The electron energy spectra fitted with a relativistic Maxwellian distribution showed that the electron temperatures were 0.98 and $1.53 \mathrm{MeV}$ for the small and large plasmas, respectively. The higher temperature of the hot electrons from the large scale preformed plasma indicates that the laser may have undergone selffocusing.

The angular distributions of hot electrons from the small and large scale preformed plasmas are rather different, as shown in Figs. 4(a) and 4(b), respectively. The hot electrons from the small preformed plasma show that the electron emission peaks between the laser axis and the target rear normal, with a beam divergence of $\sim 70^{\circ}$ (FWHM). This divergence angle is comparable to that from a solid target. ${ }^{26}$

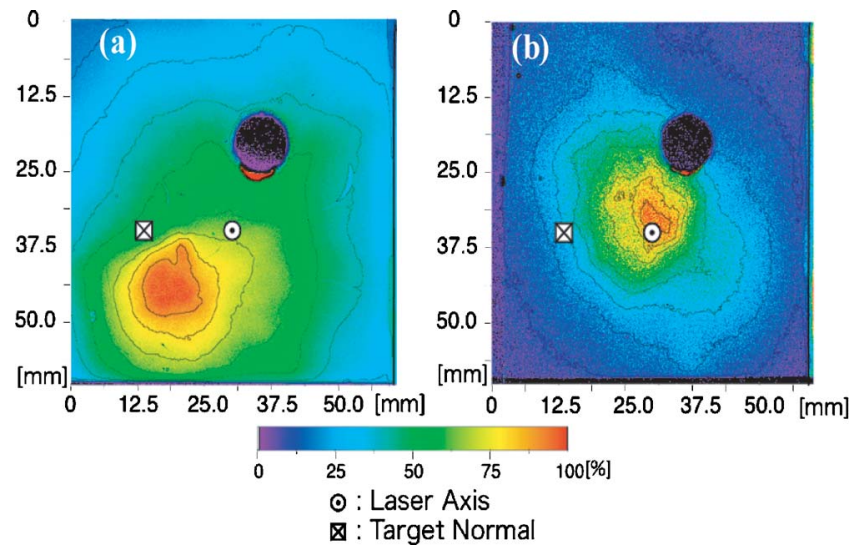

FIG. 4. (Color online) Hot electron angular distribution for (a) the small scale and (b) the large scale preformed plasma (from Ref. 24).

For the large scale preformed plasma, the hot electron emission peaks along the laser axis and has a beam divergence of $\sim 30^{\circ}$ (FWHM), which is comparable to the hard x-ray emission from ultraintense laser interaction with an imploded shell plasma. ${ }^{3}$ The on-axis electron emission indicates that the laser propagation in the large scale plasma is along its axis. This on-axis behavior of hot electrons is also different from that of ultraintense laser interaction with preplasmas created by a laser prepulse or pedestal, where the hot electron beam direction is neither stable nor along the laser axis. ${ }^{27,28}$ Highly directional and collimated hot electron beam in dense plasmas is essential for efficient heating of the compressed tiny core plasma and is of much recent interest. ${ }^{29}$

\section{MULTIPLE SHORT PULSE LASER PROPAGATION IN OVERDENSE PLASMAS}

We have shown that ultraintense lasers can self-focus and propagate deeply in a large scale preformed plasmas and generate directional collimated hot electron beams. However, this on-axis propagation of the ultraintense laser in the large scale preformed plasma is usually unstable. For example, the propagation of the laser strongly depends on its focus position and it may not propagate into high-density regions when focused at positions other than that of the optimum.

Ultraintense laser-plasma interaction can lead to nonlinear processes such as laser filamentation and beam breakup, ${ }^{8}$ scattering and propagation instabilities, ${ }^{9}$ etc. that can reduce the laser quality and propagation distance. To avoid these, one can use multiple laser pulses instead of a single one. ${ }^{30}$ We have thus investigated the interaction of a train of short, intense laser pulses with dense plasma using two-dimensional (2D) PIC simulation. ${ }^{31}$ Circularly polarized laser pulses with Gaussian envelope $a=a_{L} \exp \left[-\left(t-t_{0}\right)^{2} / \tau^{2}\right] \exp \left[-\left(y-y_{0}\right)^{2} / w^{2}\right]$ in both the longitudinal $(x)$ and lateral $(y)$ directions are incident along the $x$ axis into a plasma layer, where $a_{L}=4$ is the laser strength, $\tau$ the pulse duration and $w=5 \mu \mathrm{m}$ the spot radius. The laser intensity is thus about $10^{19} \mathrm{~W} / \mathrm{cm}^{2}$. The laser wavelength is $\lambda=1.06 \mu \mathrm{m}$. The simulation box is $60 \lambda$ along the $x$ axis and 

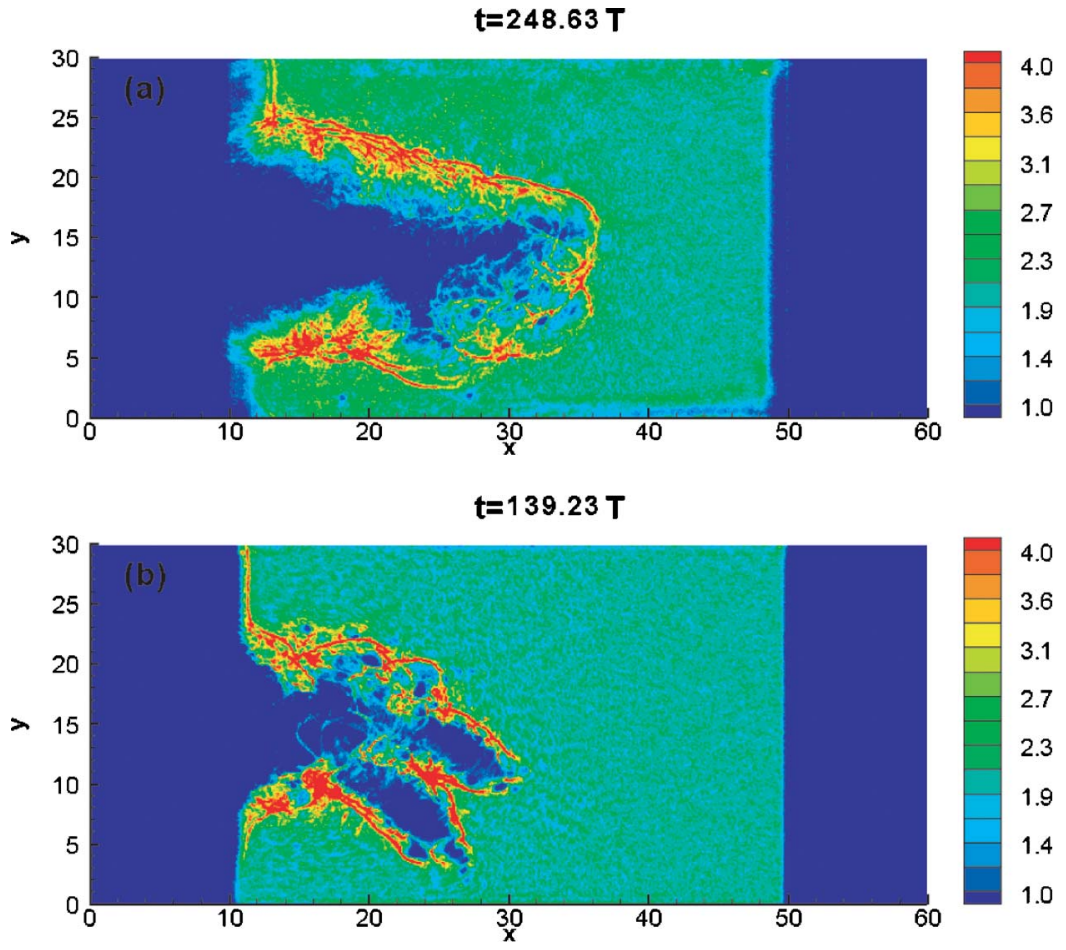

FIG. 5. (Color online) The ion density in channeling (a) by two $\tau=25 T$ pulses at $t=248.63 T$, and (b) by a single $\tau=50 T$ pulse at $t=139.23 T$, with the observation times corresponding to that at maximum channel length (from Ref. 30). The two-pulse scheme can clearly channel deeper into the plasma. The single longer (50T) pulse leads to considerable deterioration of the channel quality without lengthening it.
$30 \lambda$ along the $y$ axis. The $40 \lambda$ long uniform plasma layer of density $2 n_{c}$ is bounded by $10 \lambda$ wide vacuum regions on both its left and right sides.

We consider plasma channeling by a single $\tau=50 T$ laser pulse and two $\tau=25 T$ laser pulses, where $T=3.3$ fs is the laser period. The time delay between the peaks of the two pulses is 70T. The total energy in the two $25 T$ pulses is nearly the same as that in the $50 T$ pulse. Figure 5(b) shows the distribution of the plasma ion density for the single $\tau=50 T$ pulse at the moment when the corresponding channel extends deepest into the plasma (after the laser light has been absorbed and/or reflected). We note that the still forwardstreaming ions continue to lengthen the channel, the final channel length is reached when the forward momentum of the ions is totally spent. In Fig. 5(b), one sees that the laser is diverted in deflecting the light energy, creating a branch channel below the main one. Figure 5(a) shows the ion density for the two $\tau=25 T$ pulses at $t=248.63 T$, when the action of the second laser pulse is also over and the corresponding channel is deepest. The significant difference in the length and quality of the channels made by a single pulse and two shorter pulses indicates that the multipulse scheme is more efficient. The deeper penetration of the two-pulse laser can be attributed to the fact that the ponderomotive force acts on the plasma twice and the laser-induced plasma instabilities that can severely deteriorate the channel quality and length are greatly suppressed because of the cutoff of the source energy: Instabilities such as bending or filaments of the plasma channel caused by the first laser cannot develop much since the latter is short. When the second laser pulse then acts on the plasma at the front of the channel created by the first pulse, the plasma channel is further extended in the laser direction. Although channel branching still appears, but on smaller space scales. It is found that with suitably chosen duration and interval of the pulses, a train of short laser pulses can channel into the plasma deeper and more stably than a single longer pulse of similar peak intensity and total energy.

\section{SUMMARY}

We have investigated the propagation of intense lasers, namely, a 100 TW $0.6 \mathrm{ps}$ laser at several $10^{18} \mathrm{~W} / \mathrm{cm}^{2}$ and a 20 TW 0.6 ps laser at $10^{18} \mathrm{~W} / \mathrm{cm}^{2}$ in large scale plasmas. In the $100 \mathrm{TW}$ experiments, plasma channel formation along the laser axis was observed in a preformed plasma with $100 \mu \mathrm{m}$ density scale length and peak density $\sim 10 n_{c}$. Over $1 \mathrm{MeV}$ hot electrons with a beam divergence of about $30^{\circ}$ were emitted along the laser axis. In the 20-TW experiments, laser light transmission through preformed plasmas with peak densities of 20-50 $n_{c}$ was observed and found to depend on the laser focus position. A multiple short pulse laser propagation scheme is examined using 2D PIC simulations. Deeper and more stable (compared to the single-pulse scheme) propagation was demonstrated.

\section{ACKNOWLEDGMENTS}

We acknowledge the technical support of the engineering staff at the ILE, Osaka University, for the laser operation, target fabrication, and data acquisition.

Part of this research is supported by the JSPS JapanChina Core University Program, the Natural Science Foundation of China under the Grant Nos. 10675024, 10775165, 10835003, and 10875158, and the Science and Technology Commission of Shanghai Municipality under Grant No. 08PJ14102. 
${ }^{1}$ M. Tabak, J. Hammer, M. E. Glinsky, W. L. Kruer, S. C. Wilks, J. Woodworth, E. M. Campbell, and M. D. Perry, Phys. Plasmas 1, 1626 (1994).

${ }^{2}$ R. Kodama, P. A. Norreys, K. Mima, A. E. Dangor, R. G. Evans, H. Fujita, Y. Kitagawa, K. Krushelnick, T. Miyakoshi, N. Miyanaga, T. Norimatsu, S. J. Rose, T. Shozaki, K. Shigemori, A. Sunahara, M. Tampo, K. A. Tanaka, Y. Toyama, T. Yamanaka, and M. Zepf, Nature (London) 412, 798 (2001).

${ }^{3}$ Y. Kitagawa, Y. Sentoku, S. Akamatsu, W. Sakamoto, K. A. Tanaka, R. Kodama, H. Nishimura, Y. Inubushi, M. Nakai, T. Watari, T. Norimatsu, and A. Sunahara, Phys. Rev. E 71, 016403 (2005).

${ }^{4}$ A. B. Borisov, V. Borovskiy, V. V. Korobkin, A. M. Prokhorov, O. B. Shiryaev, X. M. Shi, T. S. Luk, A. McPherson, J. C. Solem, K. Boyer, and C. K. Rhodes, Phys. Rev. Lett. 68, 2309 (1992).

${ }^{5}$ V. A. Vshivkov, N. M. Naumova, F. Pegoraro, and S. V. Bulanov, Phys. Plasmas 5, 2727 (1998).

${ }^{6}$ S. C. Wilks, W. L. Kruer, M. Tabak, and A. B. Langdon, Phys. Rev. Lett. 69, 1383 (1992).

${ }^{7}$ A. Pukhov and J. Meyer-ter-Vehn, Phys. Rev. Lett. 79, 2686 (1997); 76, 3975 (1996)

${ }^{8}$ K. A. Tanaka, M. M. Allen, A. Pukhov, R. Kodama, H. Fujita, Y. Kato, T. Kawasaki, K. Mima, N. Morio, H. Shiraga, M. Iwata, T. Miyakoshi, and T. Yamanaka, Phys. Rev. E 62, 2672 (2000).

${ }^{9}$ Z. Najmudin, K. Krushelnick, M. Tatarakis, E. L. Clark, C. N. Danson, V. Malka, D. Neely, M. I. K. Santala, and A. E. Dangor, Phys. Plasmas 10, 438 (2003).

${ }^{10}$ M. Borghesi, A. J. MacKinnon, L. Barringer, R. Gaillard, L. A. Gizzi, C. Meyer, O. Willi, A. Pukhov, and J. Meyer-ter-Vehn, Phys. Rev. Lett. 78, 879 (1997).

${ }^{11}$ M. Zepf, M. Castro-Colin, D. Chambers, S. G. Preston, J. S. Wark, J. Zhang, C. N. Danson, D. Neely, P. A. Norreys, A. E. Dangor, A. Dyson, and P. Lee, Phys. Plasmas 3, 3242 (1996).

${ }^{12}$ J. Fuchs, J. C. Adam, F. Amiranoff, S. D. Baton, P. Gallant, A. Héron, J. C. Kieffer, G. Laval, J. L. Miquel, P. Mora, H. Pépin, and C. Rousseaux, Phys. Rev. Lett. 80, 2326 (1998).

${ }^{13}$ O. Willi, D. H. Campbell, A. Schiavi, M. Borghesi, M. Galimberti, L. A. Gizzi, W. Nazarov, A. J. Mackinnon, A. Pukhov, and J. Meyer-ter-vehn, Laser Part. Beams 19, 5 (2001).

${ }^{14}$ K. A. Tanaka, R. Kodama, H. Fujima, M. Heya, N. Izumi, Y. Kato, Y. Kitagawa, K. Mima, N. Miyanaga, T. Norimatsu, A. Pukhov, A. Sunahara, K. Takahashi, M. Allen, H. Habara, T. Habara, T. Iwatani, T. Matusita, T. Miyakosi, M. Mori, H. Setoguchi, T. Sonomoto, K. Suzuki, H. Yoshida, T. Yamanaka, Y. Sentoku, F. Weber, T. W. Barbee, Jr., and L. Da Silva, Phys. Plasmas 7, 2014 (2000).

${ }^{15}$ R. Kodama, K. Mima, K. A. Tanaka, Y. Kitagawa, H. Fujita, K. Takahashi, A. Sunahara, K. Fujita, H. Habara, T. Jitsuno, Y. Sentoku, T. Matsushita, T. Miyakoshi, N. Miyanaga, T. Norimatsu, H. Setoguchi, T. Sonomoto, M. Tanpo, Y. Toyama, and T. Yamanaka, Phys. Plasmas 8, 2268 (2001).

${ }^{16}$ Y. Kitagawa, H. Fujita, R. Kodama, H. Yoshida, S. Matsuo, T. Jitsuno, T. Kawasaki, H. Kitamura, T. Kanabe, S. Sakabe, K. Shigemori, N.
Miyanaga, and Y. Izawa, IEEE J. Quantum Electron. 40, 281 (2004).

${ }^{17}$ Y. Kitagawa, R. Kodama, K. Takahashi, M. Mori, M. Iwata, S. Tuji, K. Suzuki, K. Sawai, K. Hamada, K. Tanaka, H. Fujita, T. Kanabe, H. Takabe, H. Habara, Y. Kato, and K. Mima, Fusion Eng. Des. 44, 261 (1999).

${ }^{18}$ A. L. Lei, A. Pukhov, R. Kodama, T. Yabuuchi, K. Adumi, K. Endo, R. R. Freeman, H. Habara, Y. Kitagawa, K. Kondo, G. R. Kumar, T. Matsuoka, K. Mima, H. Nagatomo, T. Norimatsu, O. Shorokhov, R. Snavely, X. Q. Yang, J. Zheng, and K. A. Tanaka, Phys. Rev. E 76, 066403 (2007).

${ }^{19}$ H. Takabe, M. Yamanaka, K. Mima, C. Yamanaka, H. Azechi, N. Miyanaga, M. Nakatsuka, T. Jitsuno, T. Norimatsu, M. Takagi, H. Nishimura, M. Nakai, T. Yabe, T. Sasaki, K. Yoshida, K. Nishihara, Y. Kato, Y. Izawa, T. Yamanaka, and S. Nakai, Phys. Fluids 31, 2884 (1988).

${ }^{20}$ R. Kodama, K. Takahashi, K. A. Tanaka, Y. Kato, K. Murai, F. Weber, T. W. Barbee, and L. B. Da Silva, Rev. Sci. Instrum. 70, 543 (1999).

${ }^{21}$ K. Adumi, K. A. Tanaka, T. Matsuoka, T. Kurahashi, T. Yabuuchi, Y. Kitagawa, R. Kodama, K. Sawai, and K. Suzuki, Phys. Plasmas 11, 3721 (2004).

${ }^{22}$ T. Matsuoka, A. Lei, T. Yabuuchi, K. Adumi, J. Zheng, R. Kodama, K. Sawai, K. Suzuki, Y. Kitagawa, T. Norimatsu, K. Nagai, H. Nagatomo, Y. Izawa, K. Mima, Y. Sentoku, and K. A. Tanaka, Plasma Phys. Controlled Fusion 50, 105011 (2008).

${ }^{23}$ Y. Sentoku, W. Kruer, M. Matsuoka, and A. Pukhov, Fusion Sci. Technol. 49, 278 (2006).

${ }^{24}$ T. Tanimoto, A. L. Lei, T. Yabuuchi, H. Habara, K. Kondo, R. Kodama, K. Mima, and K. A. Tanaka, J. Phys.: Conf. Ser. 112, 022095 (2008).

${ }^{25}$ K. A. Tanaka, T. Yabuuchi, T. Sato, R. Kodama, Y. Kitagawa, T. Takahashi, T. Ikeda, Y. Honda, and S. Okuda, Rev. Sci. Instrum. 76, 013507 (2005).

${ }^{26}$ A. L. Lei, K. A. Tanaka, R. Kodama, G. R. Kumar, K. Nagai, T. Norimatsu, T. Yabuuchi, and K. Mima, Phys. Rev. Lett. 96, 255006 (2006).

${ }^{27}$ M. H. Key, M. D. Cable, T. E. Cowan, K. G. Estabrook, B. A. Hammel, S. P. Hatchett, E. A. Henry, D. E. Hinkel, J. D. Kilkenny, J. A. Koch, W. L. Kruer, A. B. Langdon, B. F. Lasinski, R. W. Lee, B. J. MacGowan, A. MacKinnon, J. D. Moody, M. J. Moran, A. A. Offenberger, D. M. Pennington, M. D. Perry, T. J. Phillips, T. C. Sangster, M. S. Singh, M. A. Stoyer, M. Tabak, G. L. Tietbohl, M. Tsukamoto, K. Wharton, and S. C. Wilks, Phys. Plasmas 5, 1966 (1998).

${ }^{28}$ M. I. K. Santala, M. Zepf, I. Watts, F. N. Beg, E. Clark, M. Tatarakis, K. Krushelnick, A. E. Dangor, T. McCanny, I. Spencer, R. P. Singhal, K. W. D. Ledingham, S. C. Wilks, A. C. Machacek, J. S. Wark, R. Allott, R. J. Clarke, and P. A. Norreys, Phys. Rev. Lett. 84, 1459 (2000).

${ }^{29}$ C. T. Zhou, X. T. He, and M. Y. Yu, Phys. Plasmas 13, 092109 (2006); Appl. Phys. Lett. 90, 031503 (2007); 92, 151502 (2008).

${ }^{30}$ W. Yu, L. Cao, M. Y. Yu, H. Cai, H. Xu, X. Yang, A. Lei, K. A. Tanaka, and R. Kodama, Laser Part. Beams 27, 109 (2009).

${ }^{31}$ H. Xu, W. W. Chang, H. B. Zhuo, L. H. Chang, and Z. W. Yue, Chin. J. Comput. Phys. 19, 305 (2002). 